\title{
EVALUATION OF PYRETHROID AND BOTANICAL BARRIER INSECTICIDES AGAINST AEDES ALBOPICTUS IN THE LABORATORY AND FIELD
}

\author{
STEVEN T. SMOLEROFF, CHRISTOPHER S. BIBBS, AND RUI-DE XUE \\ Anastasia Mosquito Control District, 120 EOC Drive, St. Augustine, FL 32092
}

Subject Editor: Seth Britch

\begin{abstract}
Outdoor residual insecticide applications are useful for preventing or reducing mosquito populations at focal areas. Until recently, pyrethroids have been the only option for barrier sprays in mosquito control. In this study, three pyrethroid (Onslaught, Cyzmic CS, DeltaGard) and two botanical (Nature-Cide, Essentria IC ${ }^{3}$ ) outdoor residual insecticides were comparatively tested at low, mid, and high label rates against adult Aedes albopictus in both laboratory bioassays and field trials in St. Augustine, FL, from May-August 2017. Bioassays indicated NatureCide and Cyzmic CS

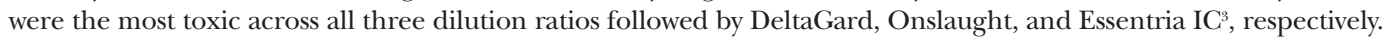
In field trials Nature-Cide and Onslaught were the only products that reduced mosquito abundance at the low rate. However, at the mid rate NatureCide and Onslaught caused $\sim 90 \%$ percent reduction of adult female Ae. albopictus in the field, the highest of all tested products. The performance of DeltaGard (79\% reduction in field counts), Essentria IC 3 (64\%), and Cyzmic CS (36\%) in the field were not similar to the laboratory results. The universally high performance of Nature-Cide indicates that mosquito control operations should expand consideration to botanical based insecticides for field operations.
\end{abstract}

Key Words: Aedes albopictus, mosquito, barrier treatments, pyrethroid, essential oils, passive control

\section{INTRODUCTION}

The Asian tiger mosquito Aedes albopictus (Skuse) is a highly invasive, peridomestic vector of arboviruses such as dengue and chikungunya (Derraik and Slaney 2015, Wilson and Chen 2015). Its adaptability and vector potential have rendered it a major public health concern while steadily increasing the global burden of vector-borne disease (Bonizzoni et al. 2013). Vector-borne diseases are responsible for more than $17 \%$ of all infectious diseases worldwide (World Health Organization 2017a). An estimated 1.38 million suspected cases of chikungunya have been recorded around the world within the last decade (World Health Organization 2017b), and during the 2016 worldwide dengue outbreak the Americas alone reported more than 2.38 million cases (World Health Organization 2017c). Targeting adult mosquito vector populations is still a key process to reduce arbovirus transmission (Manica et al. 2016).

Ground adulticide methods such as applications of a barrier treatment have com- monly been used as part of integrated mosquito management (Brown and Xue 2011). Barrier treatments are designed to stop adult mosquitoes entering areas typically used for outdoor human activity while also reducing the need to retreat the area (Fulcher et al. 2008) and treatments have been shown to be effective for focal mosquito control in these areas (Doyle et al. 2009, Brown and Xue 2011, Conover et al. 2015). Many species of adult mosquitoes such as Ae. aegypti (L.), utilize foliage structures for a variety of purposes ranging from sheltered resting sites to sources of food (Xue 2008), so barrier treatments leverage resting and feeding behaviors to maximize mosquito-insecticide contact (Fulcher et al. 2008).

Public health mosquito control in the US is restricted to only two classes of mosquito adulticide active ingredient, pyrethroids and organophosphates, which limits the options available for avoiding the evolution of resistance. For example, the majority of outdoor residual insecticides contain synthetic pyrethroid active ingredients such as bifenthrin, 
deltamethrin, sumithrin, or permethrin. Fortunately, recent work improving the emulsification of essential oils has enhanced development of plant-derived active ingredients, including synergy with existing active ingredients in adulticides and larvicides (Dias and Moraes 2013, Norris et al. 2015, Gross et al. 2017). Botanical "green" alternative insecticides are appealing due to their minimum risk classification, which allows more flexible reapplication procedures and more transparency about all ingredients in a product. To explore and evaluate available EPA exempt barrier insecticidal sprays in comparison with common pyrethroid products, we investigated the relative capabilities of three pyrethroids (type I and type II) and two botanical "green" alternative adulticides for control of adult Ae. albopictus through laboratory bioassays and field trials.

\section{MATERIALS AND METHODS}

We obtained Aedes albopictus for this study from the United States Department of Agriculture, Agricultural Research Service, Center for Medical, Agricultural, and Veterinary Entomology (CMAVE) in Gainesville, FL. Mosquitoes had been maintained in CMAVE insectaries at $26.6^{\circ} \mathrm{C}, 85 \pm 5 \%$ relative humidity (RH), $14 \mathrm{~h}$ light:10 h dark photoperiod, and fed on a $10 \%$ sucrose solution (Gerberg et al. 1994). Subjects used in bioassays were female, not blood-fed, and 6-8 days old.

We tested five barrier treatment formulations: Nature-Cide All Purpose Concentrate $(0.5 \%$ clove and $0.5 \%$ cottonseed oil; Pacific Shore Holdings, Inc., Canoga Park, CA), Essentria IC-3 (10\% rosemary, 5\% geraniol, $2 \%$ peppermint oil; Envincio LLC, Schaumberg, IL), Onslaught $(6.4 \%$ esfenvalerate, a type I pyrethroid; McLaughling Gormley King Company, Minneapolis, MN), DeltaGard (2\% deltamethrin, a type II pyrethroid; Bayer Environmental Science, Research Triangle Park, NC), and Cyzmic CS (9.7\% lambda-cyhalothrin, a type II pyrethroid; Control Solutions, Inc., Pasadena, TX). Each product was tested using label prescribed low, mid, and high application rates across separate trials.
For laboratory bioassays, we designed a cylindrical chamber using a $55 \mathrm{~mL}$ petri dish base covered with an inverted $266 \mathrm{~mL}$ (9 oz) polystyrene cup (Fig. 1). We used a hot metal probe to melt a hole through the base of the cup for aspiration and to support a sucrose solution wick, and several smaller holes around all sides of the cup for ventilation. For each of the low, mid, and high label rates, we applied $1 \mathrm{~mL}$ of formulation diluted in reverse osmosis (RO) water with a pipette to filter paper (Whatman No. 1; GE Healthcare Bio-Sciences, Pittsburgh, PA) 24 $\mathrm{h}$ in advance of bioassays. Controls consisted of $\mathrm{RO}$ water with no formulation. To begin the bioassay trials we placed treated filter papers into Petri dish bases and covered with the ventilated cups, with the cup then taped to the base as shown in Fig. 1. We introduced 15 adult female mosquitoes to each cup and fitted cotton balls saturated with $10 \%$ sucrose solution in the aspiration hole. We recorded total knockdown at $30 \mathrm{~min}$ and mortality at 24 hours. For each repetition we used 3 cups per formulation and five control

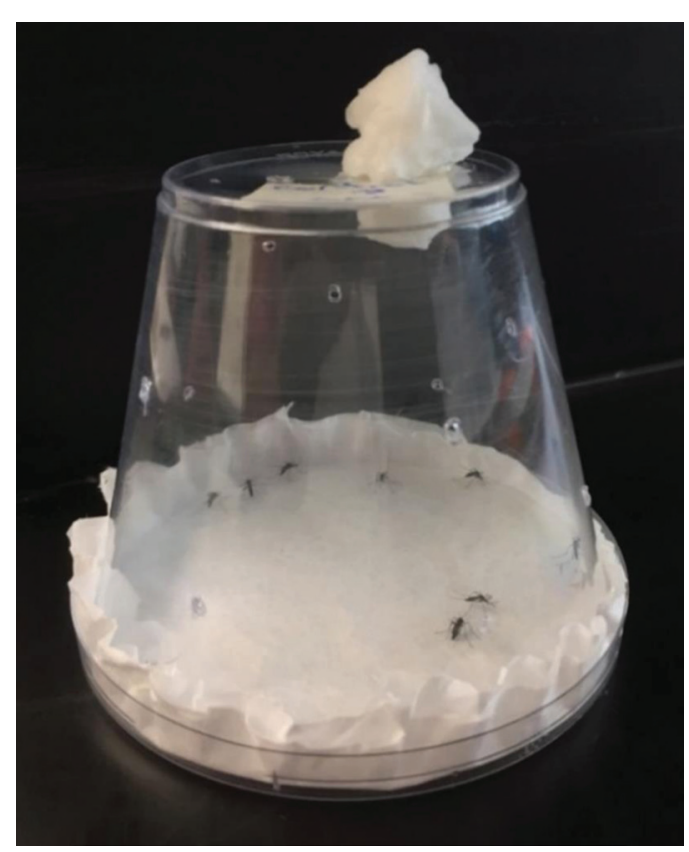

Figure 1. Bioassay chamber constructed of a Petri dish base, a pesticide-treated filter paper nested in the dish, and a ventilated polystyrene cup with sucrose solution wick, and containing 15 non-blood-fed, 5-7 d old female Aedes albopictus (Skuse). 
cups, and conducted 3 repetitions per low, mid, and high label rates.

For field tests, we selected 10 suburban sites ( 5 treatment paired with 5 control) in St. Augustine, FL, similar to the one shown in Fig. 2 based on the presence of harborage suitable for Ae. albopictus, with a minimum of $402 \mathrm{~m}$ between each paired treatment and control site. Each site was an average distance of $2.2 \mathrm{~km}$ from a central weather station where we recorded weekly rainfall summaries (Fig. 4) to provide context for patterns of mosquito population change across all sites. We conducted 3 weeks of pretreatment surveillance at each site using BioGents Sentinel (BGS) mosquito traps (BG-2; BioGents AG, Regensburg, Germany) baited with $\mathrm{CO}_{2}$ for $24 \mathrm{~h}$ per week to confirm presence of Ae. albopictus at all treatment and control sites. We identified collections from each trap weekly and continued surveillance in this way for the duration of the study.

We used a battery powered backpack sprayer (REC 15 ABZ; Birchmeier Sprühtechnik AG, Stetten, Switzerland) to apply the barrier treatments at the 5 sites, with the machine set to 5 bar flow pressure to achieve a $1,350 \mathrm{~mL} / \mathrm{min}$ flow rate. We delivered each treatment at an approximately $7-8 \mathrm{~km} / \mathrm{h}$ walking pace and calibrating each formulation-rate to a $450 \mathrm{~mL}$ application. Each site received separate but consecutive treatments for the low, mid, and high rates, in that order, with each rate left in place with surveillance for 4 weeks. We randomly assigned the 5 formulations to the 5 treat-

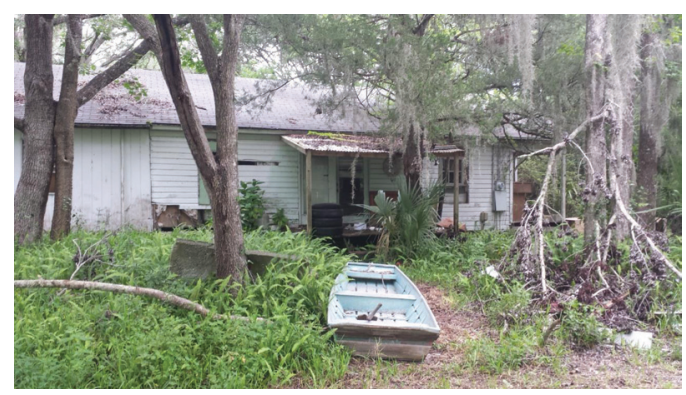

Figure. 2. Image of representative suburban field site selected based on the presence of suitable harborage for Ae. albopictus such as moderate to dense foliage, many adult resting areas, and various artificial containers for development of immature mosquitoes. ment sites, one formulation per site. Following each treatment we flushed the backpack sprayer with $3.785 \mathrm{~L}$ of water to prevent cross-contamination among formulations.

We analyzed laboratory bioassay data using an ANOVA and Tukey's HSD. For the field data, we used Mulla's formula (Mulla et al. 1971) to calculate the percent reduction in the relative abundance of wild mosquitoes as measured by adult surveillance: ${ }^{\%} \mathrm{R}=100$ $\times\left[\left(\mathrm{C}_{1} / \mathrm{T}_{1}\right) \times\left(\mathrm{T}_{2} / \mathrm{C}_{2}\right)\right] \times 100$; where $\mathrm{C}_{1}=$ pretreatment measure of mosquito abundance in the associated control site, $\mathrm{C}_{2}=$ post-treatment mosquito abundance in the control site, $\mathrm{T}_{1}=$ pre-treatment mosquito abundance in the treated site, and $\mathrm{T}_{2}=$ post-treatment mosquito abundance in the treated site. We also analyzed adult surveillance with a generalized linear model to investigate differences among treatments relative to time elapsed during the study.

\section{RESULTS AND DISCUSSION}

Results from the laboratory bioassays are summarized in Fig. 3. We found significant performance differences among the 5 formulations for both knockdown ( $\mathrm{F}=11.67$, $\mathrm{df}=4,44, P<0.0001)$ and mortality $(\mathrm{F}=$ 28.39, $\mathrm{df}=4,44, P<0.0001)$. Nature-Cide and Cyzmic CS caused the highest knockdown across all three dilution rates with $20-50 \%$ knockdown at the low rate, $100 \%$ knockdown at mid and high rates, and a mean mortality of $\geq 90 \%$ at all rates. DeltaGard, Onslaught, and Essentria $\mathrm{IC}^{3}$ had $0 \%$ knockdown and less than $20 \%$ mortality at the low rate. DeltaGard performed better at mid and high rates than Onslaught and Essentria $\mathrm{IC}^{3}$, with the latter two formulations performing poorly overall.

Analysis of field collections indicated significantly different performance among the 5 formulations $\left(\chi^{2}=10148\right.$, df $=15, P<$ $0.0001)$. Weekly changes in relative abundance of adult Ae. albopictus at field sites are shown in Fig. 4. Unfortunately, we were not able to conduct field trials at the high label rate because of limitations of time. Collections of adult female Ae. albopictus from Nature-Cide and Onslaught treat- 


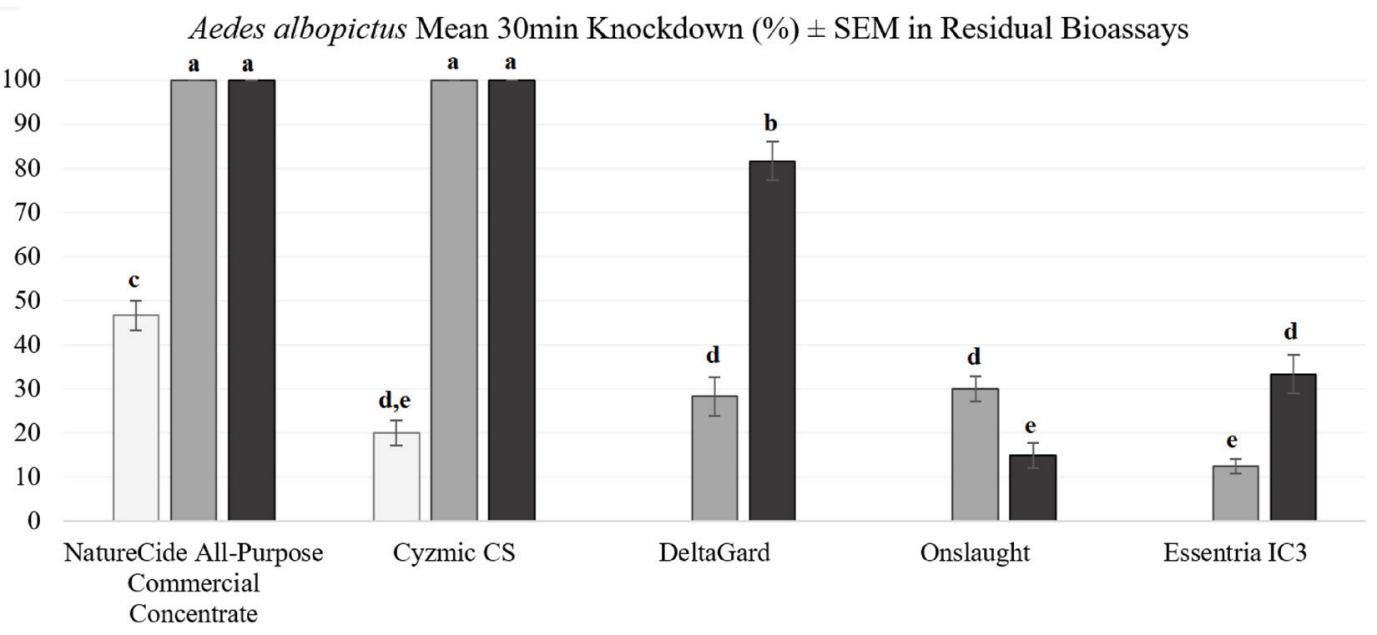

Aedes albopictus Mean 24h Mortality (\%) \pm SEM in Residual Bioassays

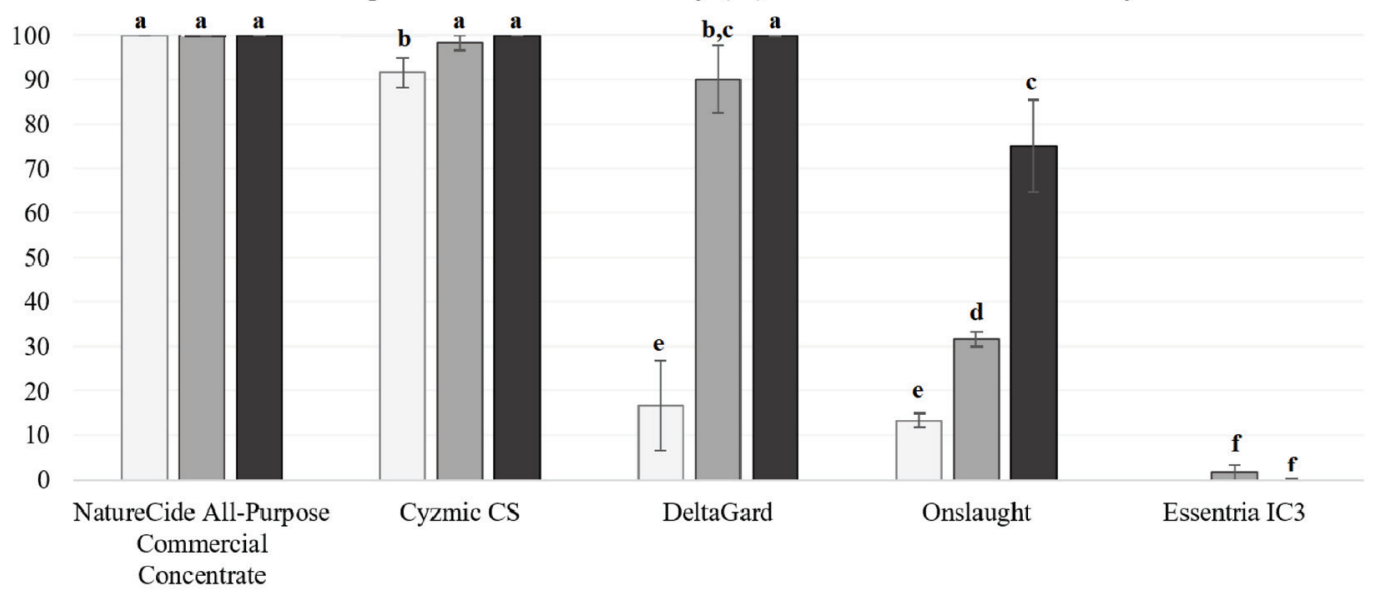

$\square$ Low $\square$ Mid $\square$ High

Figure 3. Average percent $24 \mathrm{~h}$ mortality with standard errors of the mean (ANOVA/Tukey HSD at 95\% confidence, $P<0.001$ ) of Aedes albopictus (Skuse) for each of five residual spray formulations, NatureCide All-Purpose Commercial Concentrate (clove oil, cottonseed oil; 25-100 mL/L), Cyzmic CS (lambda-cyhalothrin; 1.5-3.0 mL/L), DeltaGard (deltamethrin; 2-12 mL/L), Onslaught (fenvalerate; 4-8 mL/L), and Essentria IC; (Rosemary oil, peppermint oil; 23-47 mL/L) applied at low, mid, and high label rates. Control bioassays produced $0 \%$ mortality.

ment sites showed a net reduction of $80 \%$ by Week 8 (i.e., 4 weeks post treatment with the low rate). On the other hand, after 4 weeks with the low rate the site treated with Cyzmic had no meaningful change in relative abundance, while sites treated with DeltaGard and Essentria $\mathrm{IC}^{3}$ had a net increase in Ae. albopictus between $10 \%$ and $20 \%$. With mid-rate applications, however, sites treated with Nature-Cide and Onslaught had $90 \%$ net reductions in mosquito collections 4 weeks post treatment, compared to DeltaGard (79\% net reduction),
Essentria IC $(64 \%)$, and Cyzmic (36\%). In the GLM for the week-by-week comparison the treatment used $\left(\chi^{2}=6554.87, \mathrm{df}=5, P\right.$ $<0.0001)$ explained most of the variation, followed by the duration of weeks across the study $\left(\chi^{2}=3593.13, \mathrm{df}=10, P<0.0001\right)$.

It was surprising to find that Nature-Cide, formulated with clove and cottonseed oil as a multi-purpose insecticide, outperformed all other products in both laboratory (Fig. 3) and field (Fig. 4) trials. In contrast, the other tested botanical product, Essentria $\mathrm{IC}^{3}$, had zero to low effects 

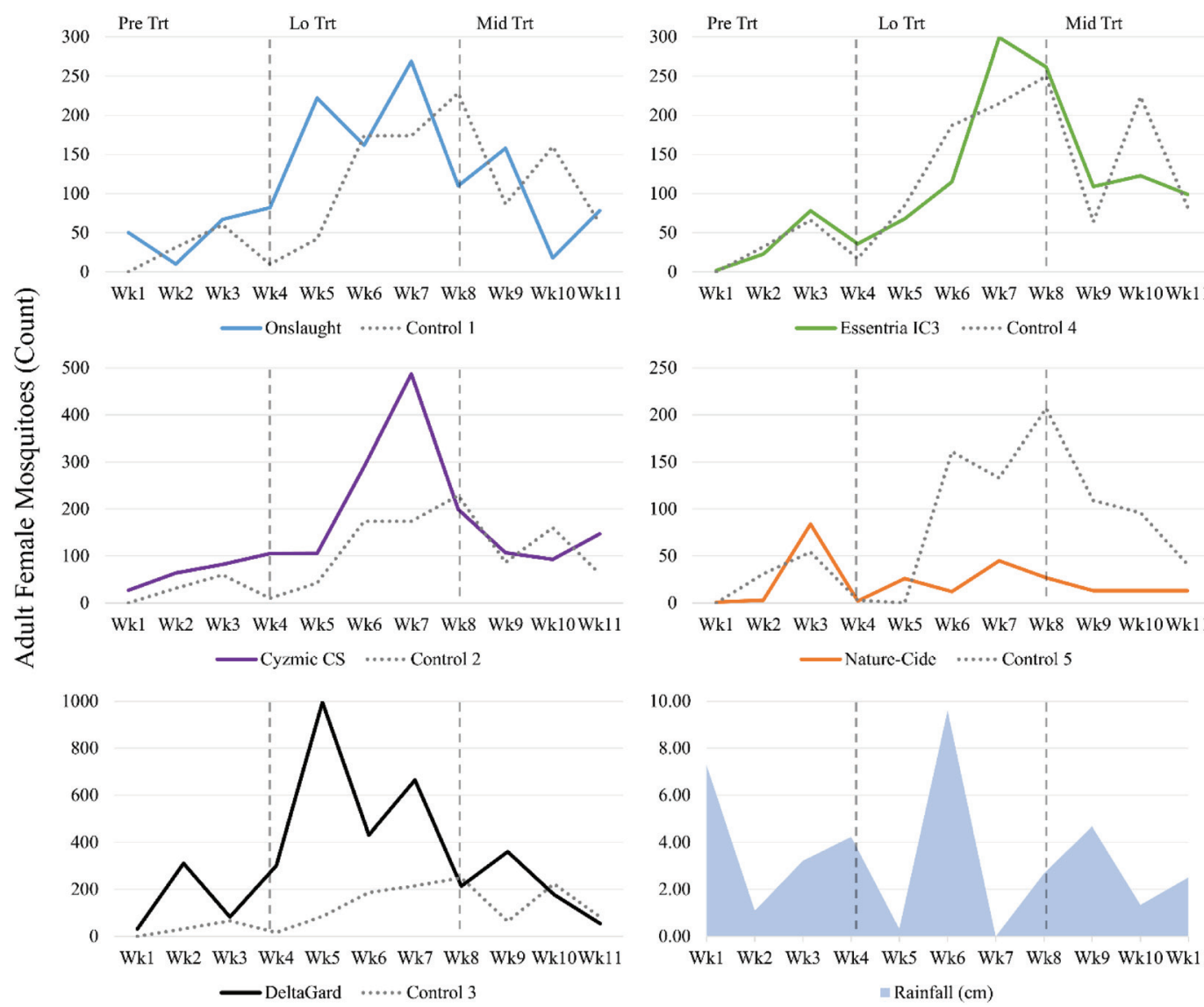

Wk1 Wk2 Wk3 Wk4 Wk5 Wk6 Wk7 Wk8 Wk9 Wk10Wk11
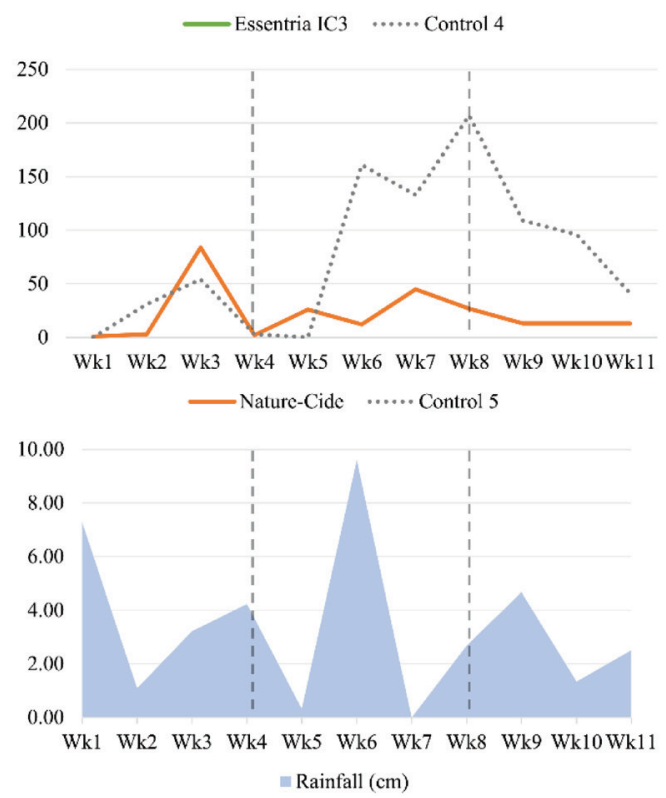

Figure 4. Field collections of adult Ae. albopictus from 5 sites treated with residual insecticides paired with 5 untreated control sites, with rainfall data $(\mathrm{cm})$ from a centrally located weather station to provide context for patterns of mosquito population change. Each graph includes results from collections for 3 weeks prior to application of the residual treatment that confirmed presence of Ae. albopictus at all treatment and control sites. We initiated experimental treatments at Week 4 with the low rate which, with the exception of Nature-Cide, did not substantially reduce Ae. albopictus abundance. We applied mid rate treatments at Week 8 resulting in Ae. albopictus reduction at all treatment sites: Nature-Cide and Onslaught ( 90\% reduction), DeltaGard ( 79\%), Essentria IC3 (64\%), and Cyzmic CS $(\sim 36 \%)$. We were not able to conduct field trials at the high label rate because of time limitations. Reduction was quantified using Mulla's formula (see text for details).

in laboratory bioassays yet low to moderate efficacy for reducing field populations of Ae. albopictus which could imply effects besides toxicity in a field environment. The rosemary, geraniol, and peppermint in Essentria $\mathrm{IC}^{3}$ could be stronger as repellents than insecticides, but we did not collect outside the treatment sites to determine if mosquito populations in adjacent areas may have increased. In comparison, the very high efficacy of Cyzmic CS, DeltaGard, and Onslaught in laboratory bioassays was not mirrored in field collections. Cyzmic CS and DeltaGard, both containing type II pyrethroids, completely failed to reduce mosquitoes when applied at the low label rate and at the mid rate performed below Onslaught, the only type I pyrethroid formulation we tested.

Pyrethroids are the most commonly used insecticides for adult mosquito control because of low environmental impact, high insecticidal potency, and good mammalian safety profiles (Amoo et al. 2008). However, the Federal, Insecticide, Fungicide, and Rodenticide Act (FIFRA) restricts the frequency that pyrethroids may be applied to the environment for adult mosquito control, spurring demand for research emphasizing green chemistry. The 
Environmental Protection Agency (EPA) allows minimum risk pesticides to be exempt from FIFRA (40 C.F.R. \$152.25 2015). Therefore, exempt pesticides containing for example the botanical ingredients described above can be applied more frequently than FIFRA labeled products. This intrinsically appeals to mosquito control programs when treatments need frequent reapplication, for example during significant mosquito outbreaks or when mitigating arbovirus transmission. Furthermore, exempt pesticides could provide different chemical classes for mosquito control programs, potentially reducing the risks of both resistance and environmental impact.

In the literature there are recent and accumulating examples of botanical oils used for mosquito control, with various ingredients functioning as repellents (Gross and Coats 2015), enhancers of other active ingredients (Gross et al. 2017), or acting as a synergist for toxicity (Tong and Bloomquist 2013, Gross et al. 2017). Plant-derived active ingredients for pesticides have generated enough interest to prompt the screening of 361 essential oils from 269 plant species as larvicides against Ae. aegypti (L.) (Dias \& Moraes 2013). Phytochemicals have also become important in adulticide development due to the success of microemulsion formulations (Montefuscoli et al. 2013, Gross et al. 2017). Commercially available plant essential oils have been screened as adulticides against Ae. aegypti and Anopheles gambiae Say with favorable results (Norris et al. 2015). Despite these impressive developments centered on plant-derived compounds for public health vector control, key botanically based products suitable for mosquito control programs such as ultra-low volume (ULV) cold aerosol space sprays are not yet developed for operational use. The positive results using Nature-Cide as an outdoor residual treatment in this study demonstrate that botanically based formulations are ready to be investigated further and possibly incorporated operationally into mosquito control programs.

\section{ACKNOWLEDGEMENTS}

We thank Jason Conrad and Univar for their assistance with selecting and sourcing the formulations chosen for this study. This is a research report only; specific mention of commercial products does not imply endorsement by the Anastasia Mosquito Control District.

\section{REFERENCES CITED}

Amoo AOJ, Xue RD, Qualls, WA, Quinn BP, Bernier UR. 2008. Residual efficacy of field-applied permethrin, d-phenothrin, and resmethrin on plant foliage against adult mosquitoes. J Am Mosq Control Assoc 24(4):543-549

Bonizzoni M, Gasperi G, Chen X, James AA. 2013. The invasive mosquito species Aedes albopictus: current knowledge and future perspectives. Trends Parasitol, 29(9):1-19.

Brown JR, Xue RD. 2011. Ground application of Aquareslin and Aquakontrol against Anopheles quadrimaculatus, Aedes aegypti, and Aedes albopictus. J Am Mosq Control Assoc 27:330-332.

Conover D, Fulcher A, Smith ML, Farooq M, Gaines MK, Xue RD. 2015. Evaluation of three commercial backpack sprayers with Aqualuer ${ }^{\circledR}$ 20-20 against caged adult Aedes aegypti. J Am Mosq Control Ass, 31(1):85-92.

Derraik JG, Slaney D. 2015. Notes on Zika- an emerging pathogen now present in the South Pacific. Aust NZ J Public Health 39:5-7.

Dias C, Moraes D. 2013. Essential oils and their compounds as Aedes aegypti L. (Diptera: Culicidae) larvicides: review. Parasite Research, 113:565-592.

Doyle MJ, Kline DL, Allan SA, Kaufman PE. 2009. Efficacy of residual bifenthrin applied to landscape vegetation against Aedes albopictus. J Am Mosq Control Ass 25:179-183.

Fulcher A, Farooq M, Smith, ML, Li CX, Scott JM, Thomson E, Kaufman PE, Xue RD. 2015. Evaluation of a new spraying machine for barrier treatment and penetration of bifenthrin on vegetation against mosquitos. J Am Mosq Control Ass, 3(1):85-92.

Gerberg EJ, Barnard DR, Ward RA. 1994. Manual for mosquito rearing and experimental techniques. AMCA Bulletin 5. Lawrence, KS: Allen Press.

Gross AD, Coats JR. 2015. Can green chemistry provide effective repellents? Insect Repellents Handbook (ed. M. Debboun, S.P. Frances, D.A. Strickman), pp. 7590. CRC Press and Taylor and Francis Group LLC, Boca Raton, FL.

Gross AD, Norris EJ, Kimber MJ, Bartholomay LC, Coats JR. 2017. Essential oils enhance the toxicity of permethrin against Aedes aegypti and Anopheles gambiae. Med Vet Entomol, 34:55-62

Manica M, Cobre P, Rosà R, Caputo B. 2016. Not in my backyard: effectiveness of outdoor residual spraying from hand-held sprayers against the mosquito Aedes albopictus in Rome, Italy. Pest Manag Sci, 73, 138-145.

Montefuscoli, A, J. Werdin-Gonzlez, S. Palma, A. Ferrero, and B. Fernandez. 2013. Design and develop- 
ment of aqueous nanoformulations for mosquito control. Parasit Research, 113:793-800.

Mulla MS, Norland LR, Fanara DM, Darwezeh HA, McKean DW. 1971. Control of chironomid midges in recreational lakes. JEcon Entomol, 64:300-307.

Norris EJ, Gross AD, Dunphy B, Bartholomay LC, Coats JR. 2015. Comparison of the insecticidal characteristics of commercially available plant essential oils against Aedes aegypti and Anopheles gambiae. J Med Entomol, 52:993-1002.

Tong F, Bloomquist JR. 2013. Plant essential oils affect the toxicities of carbaryl and permethrin against Aedes aegypti (Diptera: Culicidae). J Med Entomol, $50(4): 823-832$.

Wilson ME, Chen LH. 2016. Dengue: update on epidemiology. Curr Infec Dis Rep 17:457.
World Health Organization (WHO). (2017a) VectorBorne Diseases. Retrieved May 11th, 2018, from http://www.who.int/mediacentre/factsheets/ fs $387 /$ en/

World Health Organization (WHO). (2017b) Chikungunya. Retrieved July $15^{\text {th }}$, 2019, from https://www. who.int/news-room/fact-sheets/detail/chikungunya

World Health Organization (WHO). (2017c) Dengue and severe dengue. Retrieved July $15^{\text {th }}$, 2019, from https://www.who.int/en/news-room/fact-sheets/ detail/dengue-and-severe-dengue

Xue RD. 2008. Toxicity of permethrin-, malathion-, and fipronil-treated plant foliage to Aedes albopictus and Aedes aegypti. JAm Mosq Control Ass, 24(1):169171. 